\title{
A New Use for the Traverse Table
}

\author{
Pesi Sorab
}

There are occasions when it can be useful to plot bearings from objects not shown on the chart, for example a radiobeacon or a lighthouse outside the coverage of the chart in use. The traverse table may be used for this purpose in the following manner:

I. Establish the latitude and longitude of the object (radiobeacon, lighthouse, \&c.).

2. Choose a convenient latitude from which to plot the bearing and calculate the difference of latitude between it and the object.

3. With the bearing as course extract from the tables the departure corresponding to this d. lat.

4. For the middle (or mean) latitude derive the corresponding $d$. long. and apply it to the longitude of the bearing object.

5. Plot the bearing, as a position line, through the chosen latitude and the longitude thus derived.

Example. Navigating on Admiralty Chart 2454 in the vicinity of $50^{\circ} 20^{\prime} \mathrm{N}$, $2^{\circ} 50^{\circ} \mathrm{W}$., Start Point radiobeacon bore $25^{\circ}$. Other radiobeacons on the chart give too fine an angle of cut. However Casquets radiobeacon (not shown) bore $154^{\circ}$ some 40 miles distant.

Casquets (from, e.g., Reed's Nautical Almanac) $=49^{\circ} 43^{\prime} 5 \mathrm{~N} ., 2^{\circ} 22^{\prime} 5 \mathrm{~W}$.

Chosen latitude $=50^{\circ}$ i $5^{\prime} \mathrm{N}$.

D. lat. $=3 \div \div 5$.

Dep. $=15^{\prime} \cdot 3\left(\right.$ for course $\left.26^{\circ}\right)$.

D. long. $=24^{\prime} \circ\left(\right.$ for mid. lat. $\left.50^{\circ}\right)$.

Long. Casquets $=2^{\circ} 22: 5$.

+ d. long $=2^{\circ} 46^{\prime} \cdot 5 \mathrm{~W}$.

The position line is then drawn $154^{\circ} / 334^{\circ}$ through $50^{\circ} 15^{\prime} \mathrm{N} ., 2^{\circ} 4^{\prime}{ }_{5} \mathrm{~W}$.

I am grateful to J. Michael Gale, my instructor in navigation for the D. T. I. Yachtmaster's Certificate, for his encouragement in developing this method.

\section{'Martelli's Tables'}

\section{A. N. Black}

As the hitherto anonymous reviser who prepared the current G.H.A. edition of Martelli's Tables, 1 I was interested, instructed and surprised by Captain Cotter's article in the Forum ${ }^{2}$ on this subject. I was surprised that he should write of them as if they were things of the past. In fact they are available off the shelf at my local chart agents, and no doubt elsewhere, so a demand presumably exists today, and they are in current use. From some of the comments which he makes, 
and the extract from Table 5 which he reproduces, it appears that he has not seen the current edition.

I was interested that there had ever been any mystery about the forumula used. Although there is no explicit statement in the Introduction, inspection quickly reveals them to be, as Cotter says, the four-figure cosine-versine adaptation of the orthodox cosine-haversine method. This adaptation has been used in a number of tables, e.g. Burton's Tables, ${ }^{3}$ and those now included in Reed's Nautical Almanac. Martelli was evidently one of the earliest users of this adaptation, but I am not enough of a historian to know if he was the first. The reason for using versines, rather than haversines, is that the precision of the logarithmic, or cosine, part of the calculation to four figures is sufficient for normal navigation but that of the natural, or haversine, part of the calculation is not quite adequate. By using versines the rounding errors are halved, so the precision is doubled and the two parts of the calculation are better balanced.

It may be worth adding to Captain Cotter's description of the derivation of the tables that the factor $1 \cdot 08$ (of which the logarithm is 0.0334 ) which suddenly appears is presumably due to the author's obtaining Table 4 by copying from a table of Ternary Proportional Logarithms. This would have been less trouble than calculating the table afresh, and the added constant was easily allowed for in calculating Table 5. Ternary Proportional Logarithms, which were used in the reduction of Lunars, appeared in a number of nineteenth-century volumes of nautical tables.

Captain Cotter is only partly correct in stating that there may be serious error when the tables are employed in reducing sights of bodies with small azimuths. For small azimuths the precision of the tables is fully adequate. But it is true that if the sight is calculated as a longitude sight the calculated longitude will be unreliable, though a position line plotted through the calculated longitude will be correct within the normal limits of precision. If the assumed latitude is much in error it will be necessary to plot a very long position line; for this reason the Introduction recommends the user not to use the longitude method for any sight with an azimuth less than $30^{\circ}$.

Cosine-haversine methods fail at very high altitudes. Such altitudes can easily be avoided for longitude sights, but may occasionally be required for latitude, if the Sun or Moon is not observed when it is exactly on the meridian. A form of ex-meridian calculation is given in the Introduction to the tables to cover this case.

Captain Cotter points out that the azimuth calculation used is ambiguous near the prime vertical. The easy way of resolving this ambiguity is to take a compass bearing at the time of observation, and there is indeed a case for doing so to check the deviation. However a short auxiliary calculation which will resolve the ambiguity is provided in the Introduction. The theory of this is simple enough, but to preserve the 'Martelli Mystery', it is left to the reader to diagnose it.

Since Lecky's adverse criticism has been quoted, it is pertinent to say, risking a charge of blasphemy, that Lecky was not always right. His contemptuous dismissal of the Moon as an object to observe for position 4 led generations of mariners to disregard the Moon when it would have been very useful to them. The article of faith remained even after his reasons, such as they were, had been undermined by improvements in table construction. One small example of this stream of improvements is given by the diagrams for the Moon's total correction in the current edition of Martelli. 
The tables have been laid out to give a convenient pattern to the calculations, even at the cost of some repetitive tabulation. For instance, versines are effectively tabulated twice over, once in Table 2 and again in Table 3. The device of expressing versines in sexagesimal notation, as degrees and minutes, ensures that even the weariest and most harassed navigators will not try to add the wrong figures together. Possibly these are the reasons for the long-sustained popularity of these tables.

\section{REFEREN CES}

1 Martelli's Navigational Tables, Kelvin Hughes, Ltd., London.

2 Cotter, C. H. (1973). Martelli's tables. This Journal, 26, 485

3 Burton, S. M. and Cunningham, G. F. (1963). Burton's Four-Figure Navigation Tables, 4th Edition, Maritime Press, Ltd., London.

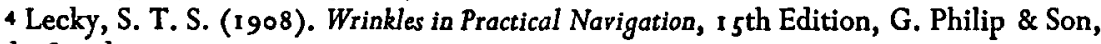
Ltd., London, p. 3 is.

\section{An Examination of Criticisms of Automatic Radar Plotting Systems and their Advantages in Relation to Manual and Semi-auto Systems}

\section{A. Harrison}

Much of Captain Wylie's note (this Journal, 27, III) is undoubtedly correct, particularly the part headed 'automatic versus manual', which restates the strong operational requirement for automatic plotting. The earlier part, however, contains some truth, some error, and shows a notable omission. A detailed analysis would take far too much space, so I will deal with three points only.

First, the opening sentence is nearly correct; I could accept it if 'supposed' is omitted, and 'plotting' reads 'predicting'. With regard to the next sentence, I must recommend a re-reading of my paper' (Capt. Wylie's ref. 3). May I re-quote my first conclusion. "The "classical" technique of manual plotting for 3 or 6 minutes gives a predicted C.P.A. whose accuracy varies within wide limits. The accuracy is adequate at long range for a fast approach, or at shorter range for a slow approach, so the method is operationally satisfactory in that adequate warning time is available in either case.'

So the criticism does state that the errors affect all methods of plotting, but goes on to say that because of differences in procedure (programme) made clear in the paper, the errors of prediction from manual plotting are acceptable in operation.

Second, the first paragraph on page $I$ I 2 implies that the errors arise only when the ship rolls, and concludes, "The steadiness of the ship contributes to a reduction of radar bearing error.' A generality; but let us, as recommended on the previous page, descend to the particular and ask 'how much?' My calculations (ref. 1) supported by experimental evidence 2.5 indicate that the error in 